\title{
NONLINEAR TIME-DEPENDENT ONE-DIMENSIONAL SCHRÖDINGER EQUATION WITH DOUBLE-WELL POTENTIAL*
}

\author{
ANDREA SACCHETTI ${ }^{\dagger}$
}

\begin{abstract}
We consider time-dependent Schrödinger equations in one dimension with doublewell potential and an external nonlinear perturbation. If the initial state belongs to the eigenspace spanned by the eigenvectors associated to the two lowest eigenvalues, then, in the semiclassical limit, we show that the reduction of the time-dependent equation to a 2-mode equation gives the dominant term of the solution with a precise estimate of the error. By means of this stability result we are able to prove the absence of the beating motion for large enough nonlinearity.
\end{abstract}

Key words. nonlinear Schrödinger operator, Gross-Pitaevskii equation, norm estimate of solutions

AMS subject classifications. 35Q40, 35B, 35K55

DOI. $10.1137 /$ S0036141002415438

1. Introduction. Recently, the theoretical analysis of the nonlinear timedependent Schrödinger equation

$$
i \hbar \dot{\psi}=H_{0} \psi+\epsilon|\psi|^{2} \psi, \quad \epsilon \in \mathrm{R}, \dot{\psi}=\frac{\partial \psi}{\partial t},
$$

where

$$
H_{0}=-\frac{\hbar^{2}}{2 m} \Delta+V, \quad \Delta=\sum_{j=1}^{d} \frac{\partial^{2}}{\partial x_{j}^{2}}, d \geq 1,
$$

has attracted an increasing interest (see [15] for a review and [11] for a rigorous derivation of the Gross-Pitaevskii energy functional). When $V$ is a double-well potential, one of the main goals is to understand how the nonlinear perturbation with strength $\epsilon$ affects the unperturbed beating motion (see, e.g., the review paper [5] and the paper [19], where (1) is proposed as a model for chiral molecules). To this end, it is crucial to study the solution $\psi$ for times of the order of the beating period; in other words, for practical purposes the unit of time is given by the beating period $T=\pi \hbar / \omega$, where $\hbar$ is the Planck's constant and $\omega$ is one-half of the energy splitting between the two lowest energies.

Here, I consider (1) in the semiclassical limit where, by assuming that $d=1$ and under some generic assumption on the double-well potential, we give the asymptotic behavior of the solution $\psi$ with a precise estimate of the error. In particular, the main result (Theorem 3) consists of proving that the solution of the Gross-Pitaevskii equation is approximated, with a rigorous control of the error, by means of the solution of an integrable two-dimensional dynamical system. As a result it follows (Theorem 4) that the beating motion between the two wells of a state initially made of the two lowest eigenstates disappears for increasing nonlinearity.

\footnotetext{
* Received by the editors October 2, 2002; accepted for publication (in revised form) May 9, 2003; published electronically January 6, 2004. This work was partially supported by the Italian MURST and INDAM-GNFM (project Comportamenti Classici in Sistemi Quantistici).

http://www.siam.org/journals/sima/35-5/41543.html

${ }^{\dagger}$ Dipartimento di Matematica, Università di Modena e Reggio Emilia, Via Campi 213/B, I-41100 Modena, Italy (sacchetti@unimo.it).
} 
A similar investigation was recently performed in [7], where the nonlinear perturbation is given by $\epsilon\langle\psi, g \psi\rangle g \psi$ and $g(x)$ is a given odd function, and in [14], where, in dimension $d=1$ and $d=3$, we consider the limit of large barrier between the two wells. In particular, in [14] I had to assume that the discrete spectrum of the Schrödinger operator $H_{0}$ consists of only two nondegenerate eigenvalues and that the restriction to the continuous eigenspace of the unitary evolution operator satisfies an a priori estimate uniformly with respect to the parameters of the model.

Finally, we mention other recent results concerning the study of the existence of stationary solutions for Gross-Pitaevskii equations with double-well potentials [2], [3] and, in the case of single-well-type potentials, the existence of solutions asymptotically given by solitary wave functions in the case when the discrete spectrum of the linear Schrödinger operator has only one nondegenerate eigenvalue [16], [21]. In the case of linear Hamiltonian $H_{0}$ with exactly two bound states Tsai and Yau [18], making use of some ideas by Soffer and Weinstein [17], proved that, in dimension $d=3$ and under certain resonance conditions, if the initial data is near a nonlinear ground state, then the solution $\psi(t, x)$ asymptotically approaches to certain nonlinear ground state.

Our paper is organized as follows.

In section 2 we introduce the main notation and state the assumptions on the potential. Moreover, we collect some semiclassical results concerning the spectrum of the linear Schrödinger operator.

In section 3 we prove the global existence of the solution of the Gross-Pitaevskii equation, the existence of conservation laws, and an a priori estimate (Theorem 2). The global existence of the solution is proved for both repulsive and attractive nonlinear perturbation, where, in the second case, we have to assume that the strength of the nonlinear perturbation is small enough.

In section 4 we introduce the two-level approximation which, roughly speaking, consists of projecting the Gross-Pitaevskii equation onto the two-dimensional space spanned by the eigenvectors of the linear Schrödinger operator associated to the two lowest eigenvalues. For practical purposes, it is more convenient to choose, as a basis of such a two-dimensional space, the two single-well states. The dynamical system we obtain is exactly solvable.

In section 5 we give our main result (Theorem 3 ) proving the stability of the twolevel approximation. Here, we make use of the comparison criterion between ordinary differential equations and an a priori estimate of the solution of the Gross-Pitaevskii equation. We emphasize that, in order to obtain such an estimate, assumption $d=1$ on the dimension plays a crucial role.

In section 6 we give the full rigorous justification of the results by Vardi [19] proving the existence of a critical value for the nonlinearity parameter giving the destruction of the beating motion (Theorem 4).

2. Assumptions and preliminary results. Here, we consider the Cauchy problem

$$
\begin{gathered}
i \hbar \dot{\psi}=H_{\epsilon} \psi, \quad H_{\epsilon}=H_{0}+W, \\
\psi(0, x)=\psi^{0}(x) \in L^{2}(\mathrm{R}), \quad\left\|\psi^{0}\right\|=1,
\end{gathered}
$$

where $\dot{\psi}$ denotes the derivative of $\psi$ with respect to the time $t, H_{0}$ is the linear Schrödinger operator formally given by (here, $x$ denotes the spatial variable in dimension 1)

$$
H_{0}=-\frac{\hbar^{2}}{2 m} \frac{d^{2}}{d x^{2}}+V
$$


$V$ is a symmetric double-well potential, and

$$
W=\epsilon|\psi|^{2}
$$

is the nonlinear perturbation with strength $\epsilon$.

In the following, for the sake of definiteness, we denote by $C$ any positive constant independent of $\epsilon, \hbar$, and $t$, we assume $\hbar$ small enough, that is, $\hbar \in\left(0, \hbar^{\star}\right]$ for some $\hbar^{\star}$, and we denote

$$
\|\varphi\|_{p}=\|\varphi\|_{L^{p}}=\left\{\int|\varphi(x)|^{p} d x\right\}^{1 / p} \text { and }\|\varphi\|=\|\varphi\|_{2} .
$$

Moreover, given $y=\left(y_{1}, \ldots, y_{m}\right) \in \mathrm{R}^{m}$ for some $m \geq 1$, we denote

$$
|y|=\max _{1 \leq j \leq m}\left|y_{j}\right| .
$$

2.1. Assumptions on the potential. Here, we assume that the potential $V$ is a regular symmetric function which admits two nondegenerate minima and it is bounded from below. More precisely, we have the following hypothesis.

HYPOTHESIS 1. The potential $V(x)$ is a real-valued function such that

(i) $V(-x)=V(x) \forall x \in \mathrm{R}$;

(ii) $V \in C^{2}(\mathrm{R})$;

(iii) $V(x)$ admits two nondegenerate minima at $x= \pm a$ for some $a>0$ such that

$$
V(x)>V_{\min }=V( \pm a) \quad \forall x \in \mathrm{R}, x \neq \pm a ;
$$

in particular, for the sake of definiteness, we assume that

$$
\frac{d V( \pm a)}{d x}=0 \quad \text { and } \quad \frac{d^{2} V( \pm a)}{d x^{2}}>0
$$

(iv) finally we assume that

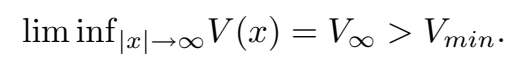

It follows that the operator formally defined in (3) admits a self-adjoint realization (still denoted by $H_{0}$ ) on $L^{2}(\mathrm{R})$ (see, for instance, Theorem III.1.1 in [4]). Let $\sigma\left(H_{0}\right)=$ $\sigma_{d} \cup \sigma_{\text {ess }}$ be the spectrum of the self-adjoint operator $H_{0}$, where $\sigma_{d}$ denotes the discrete spectrum and $\sigma_{\text {ess }}$ denotes the essential spectrum. From Hypothesis 1(iv) it follows that $\sigma_{d} \subset\left(V_{\text {min }}, V_{\infty}\right), \sigma_{\text {ess }}=\emptyset$ if $V_{\infty}=+\infty$ (see Theorem XIII.67 in [13]) and that $\sigma_{\text {ess }} \subseteq\left[V_{\infty},+\infty\right)$ if $V_{\infty}<\infty$ (see Theorem III.3.1 in [4]). Furthermore, the following two lemmas hold.

Lemma 1. Let $\sigma_{d}$ be the discrete spectrum of $H_{0}$. Then, for any $\hbar \in\left(0, \hbar^{\star}\right]$, it follows that

(i) $\sigma_{d}$ is not empty and, in particular, it contains two eigenvalues at least;

(ii) letting $\lambda_{1,2}$ be the lowest two eigenvalues of $H_{0}$, they are nondegenerate, in particular $\lambda_{1}<\lambda_{2}$, and there exists $C>0$, independent of $\hbar$, such that

$$
\inf _{\lambda \in \sigma\left(H_{0}\right)-\left\{\lambda_{1,2}\right\}}\left[\lambda-\lambda_{2}\right] \geq C \hbar .
$$

Proof. The proof is an immediate consequence of the above assumptions and standard WKB arguments. 
Lemma 2. Let $\varphi_{1,2}$ be the normalized eigenvectors associated to $\lambda_{1,2}$. Then

(i) $\varphi_{j}, j=1,2$, can be chosen to be real-valued functions such that $\varphi_{j}(-x)=$ $(-1)^{j-1} \varphi_{j}(x)$

(ii) $\varphi_{j} \in H^{1}(\mathrm{R})$;

(iii) $\varphi_{j} \in L^{p}(\mathrm{R})$ for any $p \in[1,+\infty]$;

(iv) there exists a positive constant $C$ such that

$$
\left\|\varphi_{j}\right\|_{p} \leq C \hbar^{-\frac{p-2}{4 p}} \quad \forall p \in[2,+\infty], \quad \forall \hbar \in\left(0, \hbar^{\star}\right] .
$$

Proof. Property (i) immediately follows from assumption Hypothesis 1(i). Property (ii) follows from Lemma III.3.1 in [4]. Property (iii) follows from Theorem III.3.2 in [4]. Finally, property (iv) follows for $p=+\infty$ by means of standard WKB arguments. From this fact, from the normalization of the eigenvectors, and from the Hölder inequality, property (iv) follows for any $p \in[2,+\infty]$ :

$$
\left\|\varphi_{j}\right\|_{p}=\left[\left\|\varphi_{j}^{2} \varphi_{j}^{p-2}\right\|_{1}\right]^{1 / p} \leq\left\|\varphi_{j}\right\|_{2}^{2 / p}\left\|\varphi_{j}\right\|_{\infty}^{(p-2) / p}=\left\|\varphi_{j}\right\|_{\infty}^{(p-2) / p} .
$$

2.2. Splitting and single-well states. It is well known that the splitting between the two lowest eigenvalues vanishes as $\hbar$ goes to zero. In particular, we have the following lemma.

LEMMA 3. Let

$$
\omega=\frac{\lambda_{2}-\lambda_{1}}{2} \text { and } \Omega=\frac{\lambda_{2}+\lambda_{1}}{2}
$$

and

$$
\varphi_{R}=\frac{1}{\sqrt{2}}\left[\varphi_{1}+\varphi_{2}\right] \text { and } \varphi_{L}=\frac{1}{\sqrt{2}}\left[\varphi_{1}-\varphi_{2}\right],
$$

where $\varphi_{1,2}$ are the normalized eigenvectors associated to $\lambda_{1,2}$. Then there exist two positive constants $C$ and $\Gamma$, independent of $\hbar$, such that

$$
\left\|\varphi_{R} \varphi_{L}\right\|_{\infty} \leq C \omega
$$

and

$$
\omega \leq C e^{-\Gamma / \hbar} \quad \forall \hbar \in\left(0, \hbar^{\star}\right] .
$$

As a result it follows that

$$
\lim _{\hbar \rightarrow 0} \omega=0
$$

and

$$
\lim _{\hbar \rightarrow 0} \frac{\Omega-V_{\min }}{\hbar}=c
$$

for some $c>0$.

Proof. In order to prove this lemma we observe that $V$ is a symmetric double-well potential with nonzero barrier between the wells. That is, let $\delta>0$ be small enough and let us define the two sets

$$
\left.\begin{array}{l}
B_{R}=\left\{x \in \mathrm{R}^{+}: V(x) \leq V_{\text {min }}+\delta\right\} \\
B_{L}=\left\{x \in \mathrm{R}^{-}: V(x) \leq V_{\text {min }}+\delta\right\}
\end{array}\right\}, \text { i.e., } \quad x \in B_{R} \Leftrightarrow-x \in B_{L} .
$$


From condition (5) it follows also that

$$
B_{R}=[b, c] \text { and } B_{L}=[-c,-b]
$$

for some $c>a>b>0$. The sets $B_{R, L}$ are usually called wells. Let

$$
\Gamma_{\delta}=\int_{-b}^{b} \sqrt{\max \left[V(x)-\left(V_{\min }+\delta\right), 0\right]} d x>0
$$

be the Agmon distance between the two wells. From these facts and from standard WKB arguments (see [8] and [9]) then (7)-(10) follow for some $\Gamma \in\left[\Gamma_{0}, \Gamma_{\delta}\right]$.

Remark 1. By definition it follows that $\varphi_{R}(-x)=\varphi_{L}(x)$; moreover, from (7), it follows that these functions are localized on only one of the wells $B_{R}$ and $B_{L}$; for example,

$$
\int_{B_{R}}\left|\varphi_{R}(x)\right|^{2} d x=1+O\left(e^{-C / \hbar}\right)
$$

for some $C>0$. For such a reason we call them single-well (normalized) states.

Remark 2. We emphasize that, by assuming some regularity properties on the potential $V$, it is then possible to obtain the precise asymptotic behavior of the splitting as $\hbar$ goes to zero [9].

2.3. Assumptions on the parameters. We assume that the parameter $\epsilon$ is such that

$$
\epsilon \rightarrow 0 \quad \text { as } \quad \hbar \rightarrow 0
$$

and

$$
\frac{c \epsilon}{\omega} \leq C, \quad c=\left\|\varphi_{R}^{2}\right\| \quad \forall \hbar \in\left(0, \hbar^{\star}\right]
$$

for some positive constant $C$. We recall also that the other parameter of the model, i.e., the splitting $\omega$, satisfies the asymptotic estimate (8).

\subsection{Assumption on the initial state. Let}

$$
\Pi_{c}=1-\left\langle\varphi_{R}, \cdot\right\rangle \varphi_{R}-\left\langle\varphi_{L}, \cdot\right\rangle \varphi_{L}
$$

be the projection operator onto the eigenspace orthogonal to the two-dimensional eigenspace associated to the doublet $\left\{\lambda_{1,2}\right\}$. Letting $\psi^{0}$ be the initial wave function, we assume the following.

HYPOTHESIS 2. $\Pi_{c} \psi^{0}=0$.

3. Global existence of the solution and conservation laws. Here, we prove that the Cauchy problem (2) admits a solution for all time provided that Hypotheses 1-2 are satisfied and the strength $\epsilon$ of the nonlinear perturbation is small enough. Moreover, we prove a priori estimate of the solution $\psi$.

The following results hold.

TheOREM 1. There exist $\hbar^{\star}>0$ and $\epsilon_{0}>0$ such that for any $\hbar \in\left(0, \hbar^{\star}\right]$ and $\epsilon \in\left[-\epsilon_{0}, \epsilon_{0}\right]$ the Cauchy problem (2) admits a unique solution $\psi(t, x) \in H^{1}$ for any $t \in \mathrm{R}$. Moreover, the following conservation laws hold:

$$
\|\psi(t, \cdot)\|=\left\|\psi^{0}(\cdot)\right\|=1
$$


and

$$
E(\psi)=\frac{\hbar^{2}}{2 m}\left\|\frac{\partial \psi}{\partial x}\right\|^{2}+\langle V \psi, \psi\rangle+\frac{1}{2} \epsilon\left\|\psi^{2}\right\|^{2}=E\left(\psi^{0}\right) .
$$

Proof. From Hypothesis 2 it follows that

$$
\psi^{0}=c_{1} \varphi_{1}+c_{2} \varphi_{2}, \quad c_{1,2}=\left\langle\psi^{0}, \varphi_{1,2}\right\rangle .
$$

From this fact and from Lemma $2, \psi^{0} \in H^{1}$. Therefore, existence of the global solution $\psi \in C\left(\mathrm{R}, H^{1}\right)$ and the conservation laws (13) and (14) follow from known results (see, for example, the papers quoted in [15] and [16]) for any $\epsilon>0$ (repulsive nonlinear perturbation) and for any $\epsilon \in\left(-\epsilon_{0}, 0\right)$ for some $\epsilon_{0}>0$ (attractive nonlinear perturbation).

Remark 3. There exists a positive constant $C$ independent of $\hbar$ and $\epsilon$ such that

$$
\left|E(\psi)-V_{\min }\right| \leq C\left(\omega+\hbar+\epsilon \hbar^{-1 / 2}\right) \quad \forall \hbar \in\left(0, \hbar^{\star}\right], \quad \forall \epsilon \in\left[-\epsilon_{0}, \epsilon_{0}\right] .
$$

This estimate immediately follows from (14), from Hypothesis 2, and from Lemmas 1 and 2. Indeed, from Hypothesis 2 it follows that

$$
E\left(\psi^{0}\right)=\left\langle H_{0}\left(c_{1} \varphi_{1}+c_{2} \varphi_{2}\right),\left(c_{1} \varphi_{1}+c_{2} \varphi_{2}\right)\right\rangle+\frac{1}{2} \epsilon\left\|\psi^{0}\right\|_{4}^{4},
$$

where $\left\|\psi^{0}\right\|_{4} \leq C \hbar^{-1 / 8}$ from (6) and where

$$
\left\langle H_{0}\left(c_{1} \varphi_{1}+c_{2} \varphi_{2}\right),\left(c_{1} \varphi_{1}+c_{2} \varphi_{2}\right)\right\rangle=\lambda_{1}\left|c_{1}\right|^{2}+\lambda_{2}\left|c_{2}\right|^{2}=\Omega-\omega+2 \omega\left|c_{2}\right|^{2} .
$$

From these facts and from (10), inequality (15) follows.

THEOREM 2. Let $\epsilon_{0}(\hbar)$ be a function such that

$$
\lim _{\hbar \rightarrow 0} \epsilon_{0}(\hbar) / \hbar^{2}=0 .
$$

The solution $\psi$ of (2) satisfies the following uniform estimate: there exists a positive constant $C$ independent of $t, \hbar$, and $\epsilon$ such that

$$
\|\psi\|_{p} \leq C\left[\frac{\left|E\left(\psi^{0}\right)-V_{\min }\right|}{\hbar^{2}}\right]^{\frac{p-2}{4 p}} \quad \forall p \in[2,+\infty]
$$

and

$$
\left\|\frac{\partial \psi}{\partial x}\right\| \leq C\left[\frac{\left|E\left(\psi^{0}\right)-V_{\min }\right|}{\hbar^{2}}\right]^{\frac{1}{2}}
$$

for all time and $\forall \hbar \in\left(0, \hbar^{\star}\right], \forall \epsilon \in\left[-\epsilon_{0}(\hbar), \epsilon_{0}(\hbar)\right]$.

Proof. In order to prove the estimate (17) let

$$
k=\frac{\hbar}{\sqrt{2 m}}, \quad \Lambda=\frac{E\left(\psi^{0}\right)-V_{\min }}{k^{2}} .
$$

Then the conservation laws (13) and (14) imply that

$$
\left\|\frac{\partial \psi}{\partial x}\right\|^{2}+\frac{1}{2}[\operatorname{sign}(\epsilon)] \rho^{2}\left\|\psi^{2}\right\|^{2} \leq \Lambda,
$$


where

$$
\rho=|\epsilon|^{1 / 2} / k \ll 1,
$$

according to (16). In particular, if we set

$$
\chi=\rho \psi,
$$

then the above equation takes the form

$$
\left\|\frac{\partial \chi}{\partial x}\right\|^{2}+\frac{1}{2}[\operatorname{sign}(\epsilon)]\left\|\chi^{2}\right\|^{2} \leq \Lambda \rho^{2}
$$

from which it follows that

$$
\left\|\frac{\partial \chi}{\partial x}\right\|^{2} \leq \rho^{2}|\Lambda|+\frac{1}{2}\left\|\chi^{2}\right\|^{2}=\rho^{2}|\Lambda|+\frac{1}{2}\|\chi\|_{4}^{4} .
$$

From the Gagliardo-Nirenberg inequality (see, for instance, [6] and [20], where the dimension is here equal to 1 )

$$
\|\chi\|_{2 \sigma+2}^{2 \sigma+2} \leq C\left\|\frac{\partial \chi}{\partial x}\right\|^{\sigma}\|\chi\|^{2+\sigma} \quad \forall \sigma \geq 0
$$

where we choose $\sigma=1$, it follows that

$$
\|\chi\|_{4}^{4} \leq C\left\|\frac{\partial \chi}{\partial x}\right\|\|\chi\|^{3} \leq C\left\|\frac{\partial \chi}{\partial x}\right\| \rho^{3}
$$

since $\|\chi\|=\rho\|\psi\|=\rho$ and $\|\psi\|=1$. By inserting this inequality in (18) it follows that $\left\|\frac{\partial \chi}{\partial x}\right\|$ satisfies

$$
\left\|\frac{\partial \chi}{\partial x}\right\|^{2} \leq \rho^{2}|\Lambda|+C \rho^{3}\left\|\frac{\partial \chi}{\partial x}\right\|
$$

for any $t \in \mathrm{R}$. From (20) it immediately follows that

$$
\left\|\frac{\partial \chi}{\partial x}\right\| \leq \sqrt{|\Lambda|} \rho(1+o(1)) \quad \text { as } \rho \rightarrow 0 .
$$

Hence, $\left\|\frac{\partial \psi}{\partial x}\right\| \leq C \sqrt{|\Lambda|}$ and, from (19), we have that

$$
\|\psi\|_{p} \leq C\left\|\frac{\partial \psi}{\partial x}\right\|^{\sigma / p} \leq C|\Lambda|^{(p-2) / 4 p}
$$

where we choose now $\sigma=\frac{p-2}{2}$, i.e., $p=2 \sigma+2$.

Remark 4. Condition (16) is true in the semiclassical limit and under assumption (11).

Remark 5. From the fact $E\left(\psi_{0}\right)-V_{\min }=O(\hbar)$, which follows from (8), (15), and (16), and from the bounds (17) and (11), it then follows that

$$
\|\psi\|_{p} \leq C \hbar^{-\frac{p-2}{4 p}} \quad \forall p \in[2,+\infty] \quad \text { and } \quad\left\|\frac{\partial \psi}{\partial x}\right\| \leq C \hbar^{-\frac{1}{2}}
$$

for any $t \in \mathrm{R}, \hbar \in\left(0, \hbar^{\star}\right]$, and $\epsilon \in\left[-\epsilon_{0}(\hbar), \epsilon_{0}(\hbar)\right]$. 
4. Two-level approximation. For our purposes it is more convenient to make the substitution $\psi \rightarrow e^{-i \Omega t / \hbar} \psi$; hence $(2)$ takes the following form (where, with abuse of notation, we still denote the new function by $\psi$ ):

$$
i \hbar \dot{\psi}=\left(H_{0}-\Omega\right) \psi+\epsilon|\psi|^{2} \psi, \quad \psi(x, 0)=\psi^{0}(x) .
$$

Let us write the solution of this equation in the form

$$
\psi(t, x)=a_{R}(t) \varphi_{R}(x)+a_{L}(t) \varphi_{L}(x)+\psi_{c}(t, x),
$$

where $a_{R}(t)$ and $a_{L}(t)$ are unknown complex-valued functions depending on time and $\psi_{c}=\Pi_{c} \psi, \Pi_{c}$, defined in (12), is the projection onto the space orthogonal to the two-dimensional space spanned by the two single-well states $\varphi_{R}$ and $\varphi_{L}$; i.e.,

$$
\left\langle\psi_{c}, \varphi_{R}\right\rangle=\left\langle\psi_{c}, \varphi_{L}\right\rangle=0 \quad \forall t \in \mathrm{R} .
$$

From the conservation law (13) it follows that

$$
\left|a_{R}(t)\right|^{2}+\left|a_{L}(t)\right|^{2}+\left\|\psi_{c}(t, \cdot)\right\|^{2}=1 \quad \forall t \in \mathrm{R} .
$$

By substituting $\psi$ by (23) in (2) we obtain that $a_{R}, a_{L}$, and $\psi_{c}$ must satisfy the system of differential equations

$$
\left\{\begin{array}{l}
i \hbar \dot{a}_{R}=-\omega a_{L}+\epsilon\left\langle\varphi_{R},|\psi|^{2} \psi\right\rangle \\
i \hbar \dot{a}_{L}=-\omega a_{R}+\epsilon\left\langle\varphi_{L},|\psi|^{2} \psi\right\rangle \\
i \hbar \dot{\psi}_{c}=\left(H_{0}-\Omega\right) \psi_{c}+\epsilon \Pi_{c}|\psi|^{2} \psi
\end{array}\right.
$$

By again substituting $\psi$ by (23) in the first two equations of the above system, we obtain that these equations take the form

$$
\left\{\begin{array}{l}
i \hbar \dot{a}_{R}=-\omega a_{L}+\epsilon c\left|a_{R}\right|^{2} a_{R}+\epsilon r_{R}, \\
i \hbar \dot{a}_{L}=-\omega a_{R}+\epsilon c\left|a_{L}\right|^{2} a_{L}+\epsilon r_{L}
\end{array}\right.
$$

where

$$
c=\left\|\varphi_{R}^{2}\right\|^{2}=\left\|\varphi_{L}^{2}\right\|^{2}=O\left(\hbar^{-1}\right)
$$

and where $r_{R}$ and $r_{L}$ are given by

$$
\begin{aligned}
r_{R} & =\left\langle\varphi_{R},|\psi|^{2} \psi\right\rangle-\left|a_{R}^{2}\right| a_{R}\left\langle\varphi_{R},\left|\varphi_{R}\right|^{2} \varphi_{R}\right\rangle \\
& =\left\langle\varphi_{R},|\psi|^{2} \phi_{L}\right\rangle+a_{R}\left\langle\left|\varphi_{R}\right|^{2},\left|\phi_{L}\right|^{2}+a_{R} \varphi_{R} \bar{\phi}_{L}+\bar{a}_{R} \bar{\varphi}_{R} \phi_{L}\right\rangle, \\
r_{L} & =\left\langle\varphi_{L},|\psi|^{2} \psi\right\rangle-\left|a_{L}^{2}\right| a_{L}\left\langle\varphi_{L},\left|\varphi_{L}\right|^{2} \varphi_{L}\right\rangle \\
& =\left\langle\varphi_{L},|\psi|^{2} \phi_{R}\right\rangle+a_{L}\left\langle\left|\varphi_{L}\right|^{2},\left|\phi_{R}\right|^{2}+a_{L} \varphi_{L} \bar{\phi}_{R}+\bar{a}_{L} \bar{\varphi}_{L} \phi_{R}\right\rangle,
\end{aligned}
$$

where

$$
\phi_{L}=a_{L} \varphi_{L}+\psi_{c} \quad \text { and } \quad \phi_{R}=a_{R} \varphi_{R}+\psi_{c} .
$$

We denote by two-level approximation the solutions $b_{R}$ and $b_{L}$ of the system of ordinary differential equations

$$
\left\{\begin{array}{l}
i \hbar \dot{b}_{R}=-\omega b_{L}+\epsilon c\left|b_{R}\right|^{2} b_{R}, \\
i \hbar \dot{b}_{L}=-\omega b_{R}+\epsilon c\left|b_{L}\right|^{2} b_{L},
\end{array} \quad b_{R, L}(0)=a_{R, L}(0),\right.
$$


obtained by neglecting the remainder terms $r_{R}$ and $r_{L}$ in (26). It is easy to see that the solution of this system satisfies the conservation law

$$
\left|b_{R}(t)\right|^{2}+\left|b_{L}(t)\right|^{2}=\left|b_{R}(0)\right|^{2}+\left|b_{L}(0)\right|^{2}=\left|a_{R}(0)\right|^{2}+\left|a_{L}(0)\right|^{2}=1,
$$

and, moreover, it is also possible to explicitly compute (see [12] and Appendix B in [14]) the solution of (28) by means of elliptic functions cn and dn [1]. In particular, we obtain that the imbalance function, defined as

$$
z(t)=\left|b_{R}(t)\right|^{2}-\left|b_{L}(t)\right|^{2}
$$

is given by

$$
z(t)=\left\{\begin{array}{lc}
A \operatorname{cn}\left[A \eta\left(\omega t / \hbar-\tau_{0}\right) / 2 k, k\right] & \text { if } k<1, \\
A \operatorname{dn}\left[A \eta\left(\omega t / \hbar-\tau_{0}\right) / 2,1 / k\right] & \text { if } k>1
\end{array}\right.
$$

where $\eta=\epsilon c / \omega, \tau_{0}$ depends on the initial condition,

$$
I=\sqrt{1-z^{2}(0)} \cos [\theta(0)]-\eta z^{2}(0) / 4,
$$

$\theta=\arg \left(b_{R}\right)-\arg \left(b_{L}\right)$ is the relative phase,

$$
A=\frac{2 \sqrt{2}}{\eta}\left[\sqrt{\frac{1}{4} \eta^{2}+1+I \eta}-\left(1+\frac{1}{2} I \eta\right)\right]^{1 / 2},
$$

and

$$
k^{2}=\frac{1}{2}\left[1-\frac{1+\frac{1}{2} I \eta}{\sqrt{\frac{1}{4} \eta^{2}+1+I \eta}}\right] .
$$

We emphasize that $z(t)$ periodically assumes positive and negative values if and only if $k<1$.

5. Stability of the two-level approximation. Our main result consists of proving the stability of the two-level approximation when we restore the remainder terms $r_{R}$ and $r_{L}$ in (28).

We prove the following.

TheOrem 3. Let $\psi_{c}=\Pi_{c} \psi, a_{R}(t)=\left\langle\psi, \varphi_{R}\right\rangle$, and $a_{L}(t)=\left\langle\psi, \varphi_{L}\right\rangle$, where $\psi$ is the solution of (22), and let $b_{R}(t)$ and $b_{L}(t)$ be the solution of the system of ordinary differential equations (28). Let $\epsilon \in\left[-\epsilon_{0}(\hbar), \epsilon_{0}(\hbar)\right]$, where $\epsilon_{0}(\hbar)$ satisfies the condition (16). Then, for any $\tau^{\prime}>0$, there exists a positive constant $C$ independent of $\epsilon, \hbar$, and $t$ such that

$$
\left|b_{R, L}(t)-a_{R, L}(t)\right| \leq C e^{-C \hbar^{-1}} \text { and }\left\|\psi_{c}(\cdot, t)\right\| \leq C e^{-C \hbar^{-1}}
$$

for any $\hbar \in\left(0, \hbar^{\star}\right]$ and for any $t \in\left[0, \hbar \tau^{\prime} / \omega\right]$.

Proof. For the sake of simplicity, hereafter, we omit the parameters when doing so does not cause misunderstandings. In order to prove the theorem we introduce the slow time $\tau=\omega t / \hbar$ and let

$$
\left\{\begin{array}{l}
A_{R, L}(\tau)=a_{R, L}(t), \\
B_{R, L}(\tau)=b_{R, L}(t),
\end{array} \quad R_{R, L}(\tau)=\frac{\epsilon}{\omega} r_{R, L}(t), \quad \text { and } \quad \eta=\frac{\epsilon c}{\omega} .\right.
$$


Then (26) and (28), respectively, take the form (here ' denotes the derivative with respect to $\tau)$

$$
\left\{\begin{array}{c}
A_{R}^{\prime}=i A_{L}-i \eta\left|A_{R}\right|^{2} A_{R}+R_{R}, \\
A_{L}^{\prime}=i A_{R}-i \eta\left|A_{L}\right|^{2} A_{L}+R_{L}
\end{array}\right.
$$

and

$$
\left\{\begin{array}{l}
B_{R}^{\prime}=i B_{L}-i \eta\left|B_{R}\right|^{2} B_{R} \\
B_{L}^{\prime}=i B_{R}-i \eta\left|B_{L}\right|^{2} B_{L}
\end{array}\right.
$$

satisfying the same initial condition

$$
B_{R, L}(0)=A_{R, L}(0)=a_{R, L}(0) .
$$

Due to (24) and (29), they are such that

$$
\left|B_{R}(\tau)\right|^{2}+\left|B_{L}(\tau)\right|^{2}=1, \quad\left|A_{R}(\tau)\right|^{2}+\left|A_{L}(\tau)\right|^{2} \leq 1 .
$$

In a more concise way, with an obvious meaning of notation, we can write (33) and (34) as

$$
A^{\prime}=f(A)+R \quad \text { and } \quad B^{\prime}=f(B), \quad A(0)=B(0)=a(0),
$$

where $A, B \in S^{2}$ since (35), $S^{2}=\left\{\left(z_{1}, z_{2}\right) \in \mathrm{C}^{2}:\left|z_{1}\right|^{2}+\left|z_{2}\right|^{2} \leq 1\right\}$.

Lemma 4. The function $f: S^{2} \rightarrow C^{2}$ satisfies the Lipschitz condition

$$
|f(A)-f(B)| \leq L|A-B|, L=1+3 \eta .
$$

Proof. According to the notation (4) we have

$$
|f(A)-f(B)|=\max \left[\left|f_{R}\right|,\left|f_{L}\right|\right],
$$

where $|A| \leq 1$ and $|B| \leq 1$ since $A, B \in S^{2}$, and where

$$
\begin{aligned}
& f_{R}=\left(A_{L}-B_{L}\right)-\eta\left(\left|A_{R}\right|^{2} A_{R}-\left|B_{R}\right|^{2} B_{R}\right), \\
& f_{L}=\left(A_{R}-B_{R}\right)-\eta\left(\left|A_{L}\right|^{2} A_{L}-\left|B_{L}\right|^{2} B_{L}\right) .
\end{aligned}
$$

Then (37) immediately follows since

$$
f_{R}=\left(A_{L}-B_{L}\right)-\eta\left[\left|B_{R}\right|^{2}\left(A_{R}-B_{R}\right)+A_{R}\left(\left|A_{R}\right|+\left|B_{R}\right|\right)\left(\left|A_{R}\right|-\left|B_{R}\right|\right)\right],
$$

where ||$A_{R}|-| B_{R}|| \leq\left|A_{R}-B_{R}\right|$, and where the other term $f_{L}$ will be treated the same way.

LEMMA 5. Let

$$
\beta=\max [c \epsilon, \omega],
$$

where $c$ is defined in (27). Let $\psi_{c}=\Pi_{c} \psi$, where $\psi$ is the solution of (22); it satisfies the uniform estimate

$$
\left\|\psi_{c}\right\| \leq C \beta \hbar^{-3 / 2}\left[\exp \left[C \hbar^{-1 / 2}(\epsilon t / \hbar)\right]+1\right] \quad \forall t \in \mathrm{R}
$$


for some positive constant $C$ independent of $\hbar, \epsilon$, and $t$.

Proof. As a first step we consider the following raw estimates:

$$
\left\|\psi_{c}\right\|_{p} \leq C \hbar^{-\frac{p-2}{4 p}} \quad \forall p \in[2,+\infty], \quad \forall t \in \mathrm{R}
$$

and

$$
\left|r_{R, L}\right| \leq C \hbar^{-1 / 2} \quad \forall t \in \mathrm{R} .
$$

Indeed, (39) immediately follows from the Minkowski inequality and from (21):

$$
C \hbar^{-\frac{p-2}{4 p}} \geq\|\psi\|_{p} \geq-\left(\left|a_{R}(t)\right|\left\|\varphi_{R}\right\|_{p}+\left|a_{L}(t)\right|\left\|\varphi_{L}\right\|_{p}\right)+\left\|\psi_{c}\right\|_{p},
$$

where $\left|a_{R, L}(t)\right| \leq 1$, and where $\varphi_{R, L}$ satisfy the bound (6). In the same way, from Lemma 2 and Theorem 2, it follows that

$$
\begin{aligned}
\left|r_{R}\right| & \leq C\left\|\varphi_{R} \psi^{2}\right\| \cdot\|\psi\|+\left\|\varphi_{R}\right\|_{4}^{4} \\
& \leq C\left\|\varphi_{R}\right\|_{\infty}\|\psi\|_{4}^{2}\|\psi\|+C\left\|\varphi_{R}\right\|_{4}^{4} \\
& \leq C \hbar^{-1 / 2},
\end{aligned}
$$

and similarly for $\left|r_{L}\right|$.

Now, in order to prove the estimate (38) we make use of the third equation of (25), from which it follows that

$$
\psi_{c}(\cdot, t)=-i \frac{\epsilon}{\hbar} \int_{0}^{t} e^{-i\left(H_{0}-\Omega\right)(t-s) / \hbar} \Pi_{c}|\psi(\cdot, s)|^{2} \psi(\cdot, s) d s
$$

since $\psi_{c}^{0}=\Pi_{c} \psi^{0}=0$ from Hypothesis 2 .

Let $\psi=\varphi+\psi_{c}$, where $\varphi=a_{R} \varphi_{R}+a_{L} \varphi_{L}$. Then

$$
|\psi|^{2} \psi=\varphi_{I}+\psi_{c} \varphi_{I I}+\bar{\psi}_{c} \varphi_{I I I},\left\{\begin{array}{l}
\varphi_{I}=|\varphi|^{2} \varphi \\
\varphi_{I I}=2|\varphi|^{2}+2 \bar{\psi}_{c} \varphi+\left|\psi_{c}\right|^{2}+\bar{\varphi} \psi_{c} \\
\varphi_{I I I}=\varphi^{2}
\end{array}\right.
$$

Therefore, we can write

$$
\psi_{c}=-i \frac{\epsilon}{\hbar}[I+I I+I I I]
$$

where

$$
\begin{gathered}
I=\int_{0}^{t} e^{-i\left(H_{0}-\Omega\right)(t-s) / \hbar} \Pi_{c} \varphi_{I} d s, \\
I I=\int_{0}^{t} e^{-i\left(H_{0}-\Omega\right)(t-s) / \hbar} \Pi_{c} \psi_{c} \varphi_{I I} d s, \\
I I I=\int_{0}^{t} e^{-i\left(H_{0}-\Omega\right)(t-s) / \hbar} \Pi_{c} \bar{\psi}_{c} \varphi_{I I I} d s .
\end{gathered}
$$

For the first term we have, by integrating by parts, that

$$
\begin{aligned}
I= & {\left[-i \hbar e^{-i\left(H_{0}-\Omega\right)(t-s) / \hbar}\left[H_{0}-\Omega\right]^{-1} \Pi_{c}|\varphi|^{2} \varphi\right]_{0}^{t} } \\
& +i \hbar \int_{0}^{t} e^{-i\left(H_{0}-\Omega\right)(t-s) / \hbar}\left[H_{0}-\Omega\right]^{-1} \Pi_{c} \frac{\partial|\varphi|^{2} \varphi}{\partial s} d s .
\end{aligned}
$$


Let us emphasize that from Lemma 1 it follows that the following operators, from $L^{2}$ into $L^{2}$, are bounded:

$$
\left\|e^{-i\left(H_{0}-\Omega\right)(t-s) / \hbar}\right\|=1, \quad\left\|\hbar\left[H_{0}-\Omega\right]^{-1} \Pi_{c}\right\| \leq C .
$$

Also, from Lemma 2 and (24), (26), and (27), we have the following uniform estimate for any $t \in \mathrm{R}$ :

$$
\begin{aligned}
\|\dot{\varphi}\|_{p} & \leq\left(\left|\dot{a}_{r}\right|+\left|\dot{a}_{L}\right|\right)\left(\left\|\varphi_{R}\right\|_{p}+\left\|\varphi_{L}\right\|_{p}\right) \leq C \hbar^{-1} \max \left[c \epsilon, \omega, \epsilon \hbar^{-\frac{1}{2}}\right] \hbar^{-\frac{p-2}{4 p}} \\
& \leq C \hbar^{-1} \beta \hbar^{-\frac{p-2}{4 p}} .
\end{aligned}
$$

Then we have that

$$
\begin{aligned}
\|I\| & \leq C \max _{s \in[0, t]}\left\{\left\|\varphi^{3}(s, \cdot)\right\|+t\left\|\dot{\varphi}(s, \cdot) \varphi^{2}(s, \cdot)\right\|\right\} \\
& \leq C \max _{s \in[0, t]}\left\{\|\varphi(s, \cdot)\|_{6}^{3}+t\|\dot{\varphi}(s, \cdot)\| \cdot\|\varphi(s, \cdot)\|_{\infty}^{2}\right\} \\
& \leq C\left\{\hbar^{-1 / 2}+t \hbar^{-1} \beta \hbar^{-1 / 2}\right\} .
\end{aligned}
$$

For the other two terms we have that

$$
\|I I\| \leq \int_{0}^{t}\left\|\psi_{c}\right\| \cdot\left\|\varphi_{I I}\right\|_{\infty} d s \leq C \hbar^{-1 / 2} \int_{0}^{t}\left\|\psi_{c}\right\| d s
$$

since $\left\|\varphi_{I I}\right\|_{\infty} \leq C \hbar^{-1 / 2}$, and similarly

$$
\|I I I\| \leq \int_{0}^{t}\left\|\psi_{c}\right\| \cdot\left\|\varphi_{I I I}\right\|_{\infty} d s \leq C \hbar^{-1 / 2} \int_{0}^{t}\left\|\psi_{c}\right\| d s .
$$

Indeed, from Lemma 2 and (39) it follows that

$$
\left\|\varphi_{I I}\right\|_{\infty} \leq C\left\{\|\varphi\|_{\infty}^{2}+\left\|\psi_{c}\right\|_{\infty}\|\varphi\|_{\infty}+\left\|\psi_{c}\right\|_{\infty}^{2}\right\} \leq C \hbar^{-1 / 2}
$$

and

$$
\left\|\varphi_{I I I}\right\|_{\infty} \leq\|\varphi\|_{\infty}^{2} \leq C \hbar^{-1 / 2} .
$$

Collecting all these results and denoting

$$
g(t)=\left\|\psi_{c}(\cdot, t)\right\|
$$

we have that $g(t)$ is a positive real-valued function satisfying the estimate

$$
\begin{aligned}
g(t) & \leq C \frac{\epsilon}{\hbar}\left\{\hbar^{-1 / 2} \int_{0}^{t} g(s) d s+\hbar^{-1 / 2}\left(1+t \hbar^{-1} \beta\right)\right\} \\
& \leq a \int_{0}^{t} g(s) d s+a+a b t, \quad a=C \frac{\epsilon}{\hbar^{3 / 2}}, \quad b=\frac{\beta}{\hbar} .
\end{aligned}
$$

From this estimate, since $\psi_{c}(0)=0$, and from Gronwall's lemma (see [10], page 19) it follows that

$$
\begin{aligned}
g(t) & \leq a+a b t+a \int_{0}^{t} e^{a(t-s)}(a+a b s) d s=-b+a e^{a t}+b e^{a t} \\
& \leq \frac{C \beta}{\hbar^{3 / 2}}\left[e^{C \epsilon t \hbar^{-3 / 2}}+1\right],
\end{aligned}
$$


proving the result.

From the inequality (8) and from assumption (11) it follows that for any fixed $\tau^{\prime}>0$ there exists $C>0$ satisfying the second inequality in (32).

Lemma 6. For any fixed $\tau^{\prime}>0$ the remainder terms $r_{R}$ and $r_{L}$ satisfy the uniform estimate

$$
\max \left[\left|r_{R}\right|,\left|r_{L}\right|\right] \leq C \beta \hbar^{-2} e^{C \hbar^{-1 / 2}} \quad \forall t \in\left[0, \tau^{\prime} \hbar / \omega\right]
$$

for some positive constant $C$ independent of $\hbar, \epsilon$, and $t$.

Proof. Let us consider only the term $\left|r_{R}\right|$; the other term $\left|r_{L}\right|$ could be treated the same way. By definition, and since $\max \left[\left|a_{R}\right|,\left|a_{L}\right|\right] \leq 1$, it follows that

$$
\begin{aligned}
\left|r_{R}\right| \leq & +\left|\left\langle\varphi_{R} \bar{\varphi}_{L},|\psi|^{2}\right\rangle\right| \\
& +\left|\left\langle\varphi_{R}|\psi|^{2}, \psi_{c}\right\rangle\right| \\
& +\left|\left\langle\left|\varphi_{R}\right|^{2},\left|\phi_{L}\right|^{2}+a_{R} \varphi_{R} \bar{\phi}_{L}+\bar{a}_{R} \bar{\varphi}_{R} \phi_{L}\right\rangle\right|,
\end{aligned}
$$

and we estimate separately each term.

From Lemma 3, equation (13), and the Hölder inequality, it follows that the term (40) satisfies the estimate

$$
\left|\left\langle\varphi_{R} \varphi_{L},|\psi|^{2}\right\rangle\right| \leq\left\|\varphi_{R} \bar{\varphi}_{L}\right\|_{\infty} \cdot\left\|\psi^{2}\right\|_{1} \leq C \omega .
$$

From Lemma 5 and the Hölder inequality, it follows that the term (41) satisfies the estimates

$$
\left|\left\langle\varphi_{R}|\psi|^{2}, \psi_{c}\right\rangle\right| \leq\left\|\varphi_{R}\right\|_{\infty} \cdot\left\|\psi^{2}\right\| \cdot\left\|\psi_{c}\right\| \leq C \beta \hbar^{-2} e^{C \hbar^{-1 / 2}}
$$

and that the term (42) satisfies the estimate

$$
\begin{aligned}
& \left|\left\langle\left|\varphi_{R}\right|^{2},\left|\phi_{L}\right|^{2}+a_{R} \varphi_{R} \bar{\phi}_{L}+\bar{a}_{R} \bar{\varphi}_{R} \phi_{L}\right\rangle\right| \\
& \quad \leq C\left[\left\|\varphi_{R} \varphi_{L}\right\|_{\infty}+\left\|\varphi_{R}^{2}\right\|_{\infty}\left\|\psi_{c}\right\|^{2}+\left\|\varphi_{R} \varphi_{L}\right\|_{\infty}\left\|\psi_{c}\right\|\right] \leq C \omega .
\end{aligned}
$$

Collecting all these estimates, we obtain the proof of the lemma.

The proof of the theorem is almost complete. Indeed, equations (36) can be rewritten in the integral form

$$
A(\tau)=A(0)+\int_{0}^{\tau} f[A(s)] d s+\int_{0}^{\tau} R d s
$$

and

$$
B(\tau)=B(0)+\int_{0}^{\tau} f[B(s)] d s
$$

from which, and from Lemmas 4 and 5 , it follows that for any $\tau \in\left[0, \tau^{\prime}\right]$,

$$
\begin{aligned}
|A(\tau)-B(\tau)| & \leq \int_{0}^{\tau}|f[A(s)]-f[B(s)]| d s+\int_{0}^{\tau}|R| d s \\
& \leq a \int_{0}^{\tau}|A(s)-B(s)| d s+b \tau, \quad a=L, \quad b=C \frac{\epsilon \beta \hbar^{-2} e^{C \hbar^{-1 / 2}}}{\omega} .
\end{aligned}
$$


From this inequality and by means of Gronwall's lemma we finally obtain that

$$
\begin{aligned}
|A(\tau)-B(\tau)| & \leq b \tau+a b \int_{0}^{\tau} e^{a(\tau-s)} s d s=\frac{b}{a}\left[e^{a \tau}-1\right] \\
& \leq \frac{C}{L} \frac{\epsilon \beta \hbar^{-2} e^{C \hbar^{-1 / 2}}}{\omega}
\end{aligned}
$$

proving (32) since

$$
\frac{\omega+\epsilon}{C^{\prime} \omega} \leq L=1+3 \eta \leq C^{\prime} \frac{\omega+\epsilon}{\omega}
$$

for some $C^{\prime}>0$, which implies that $\frac{\beta}{L \omega} \leq C$ for some $C>0$.

Remark 6 . Since $\omega=O\left(e^{-\Gamma / \hbar}\right)$ the above theorem implies that for any $\alpha<1$ and for any $\tau^{\prime}>0$, there exists $C$ such that

$$
\left|b_{R, L}(t)-a_{R, L}(t)\right| \leq C \omega^{\alpha} \quad \text { and } \quad\left\|\psi_{c}(\cdot, t)\right\| \leq C \omega^{\alpha} \quad \forall t \in\left[0, \hbar \tau^{\prime} / \omega\right] .
$$

\section{Destruction of the beating motion for large nonlinearity.}

6.1. The unperturbed case $\epsilon=\mathbf{0}$. Under Hypothesis 2 it follows that the solution of the unperturbed equation

$$
i \hbar \dot{\psi}=H_{0} \psi, \quad \psi(0, x)=\psi^{0}(x)
$$

is simply given by

$$
\begin{aligned}
\psi(t, x)= & e^{-i \Omega t / \hbar}\left[\frac{c_{1}+c_{2}}{\sqrt{2}} \cos (\omega t / \hbar)+i \frac{c_{2}-c_{1}}{\sqrt{2}} \sin (\omega t / \hbar)\right] \varphi_{R}(x) \\
& +e^{-i \Omega t / \hbar}\left[\frac{c_{1}-c_{2}}{\sqrt{2}} \cos (\omega t / \hbar)-i \frac{c_{1}+c_{2}}{\sqrt{2}} \sin (\omega t / \hbar),\right] \varphi_{L}(x),
\end{aligned}
$$

where

$$
c_{1,2}=\left\langle\varphi_{1,2}, \psi^{0}\right\rangle, \quad\left|c_{1}\right|^{2}+\left|c_{2}\right|^{2}=1
$$

Hence, $\psi(t, x)$ is, up to the phase factor $e^{-i(\Omega-\omega) t / \hbar}$, a periodic function with period $T=\pi \hbar / \omega$.

In particular, if $\psi$ initially coincides with a single-well state, e.g., $\psi^{0}=\varphi_{R}$, then

$$
\psi(t, x)=e^{-i(\Omega-\omega) t / \hbar}\left[e^{-i \omega t / \hbar} \cos (\omega t / \hbar) \varphi_{R}(x)-i e^{-i \omega t / \hbar} \sin (\omega t / \hbar) \varphi_{L}(x)\right]
$$

and the state $\psi(t, x)$ performs a beating motion. That is, the state, initially localized on the well $B_{R}$, is localized on the other well $B_{L}$ after half a period and, after a whole 
period, it returns to the initial well, and so on. In particular, let us consider the motion of the center of mass defined here as

$$
\langle X\rangle^{t}=\langle X \psi, \psi\rangle=\int_{\mathrm{R}} X(x)|\psi(t, x)|^{2} d x,
$$

where $X \in C(R) \cap L^{2}(\mathrm{R})$ is a given bounded function such that $X(-x)=-X(x)$. We have that

$$
\langle X\rangle^{t}=X_{0}\left[\cos ^{2}(\omega t / \hbar)-\sin ^{2}(\omega t / \hbar)\right]
$$

where

$$
X_{0}=\left\langle\varphi_{R}, X \varphi_{R}\right\rangle=\int_{\mathrm{R}} X(x)\left|\varphi_{R}(x)\right|^{2} d x .
$$

Hence, $\langle X\rangle^{t}$ is a periodic function which periodically assumes positive and negative values; i.e., we have the well-known beating motion for the double-well problem.

6.2. The perturbed case $\boldsymbol{\epsilon} \neq \mathbf{0}$. In such a case it follows that the center of mass is given by

$$
\langle X\rangle^{t}=X_{0}\left[\left|a_{R}(t)\right|^{2}-\left|a_{L}(t)\right|^{2}\right]+r,
$$

where $X_{0}$ has been previously defined and the remainder term $r$ satisfies the uniform estimate

$$
\begin{aligned}
|r| & =2\left|\Re\left[a_{R} \bar{a}_{L}\left\langle X \varphi_{R}, \varphi_{L}\right\rangle+\left\langle X \psi, \psi_{c}\right\rangle\right]\right| \\
& \leq 2\left[\left\|\varphi_{R} \varphi_{L}\right\|_{\infty}+\|X\|_{\infty}\|\psi\|\left\|\psi_{c}\right\|\right] \\
& \leq C e^{-C \hbar^{-1}} \quad \forall t \in\left[0, \hbar \tau^{\prime} / \omega\right] .
\end{aligned}
$$

If we denote by $z(t)$ the imbalance function defined in (30), then, in the semiclassical limit, it follows that

$$
\left|a_{R}(t)\right|^{2}-\left|a_{L}(t)\right|^{2} \sim z(t) \quad \forall t \in\left[0, \hbar \tau^{\prime} / \omega\right]
$$

hence

$$
\langle X\rangle^{t} \sim X_{0} z(t) \quad \forall t \in\left[0, \hbar \tau^{\prime} / \omega\right] .
$$

Then we have the following.

TheOREM 4. Let Hypotheses 1 and 2 be satisfied. Let $k^{2}$ be defined as in (31), depending on the initial wave function $\psi^{0}$. Let $\tau^{\prime}>0$ be fixed, and let $\langle X\rangle^{t}$, up to a remainder term, be a periodic function for any $t \in\left[0, \hbar \tau^{\prime} / \omega\right]$. In particular, if

(i) $k^{2}<1$, then $\langle X\rangle^{t}$ periodically assumes positive and negative values (i.e., the beating motion still persists);

(ii) $k^{2}>1$, then $\langle X\rangle^{t}$ has a definite sign (i.e., the beating motion is forbidden).

Remark 7 . Let us close by emphasizing that when the wave function is initially prepared on just one well, e.g., $\psi^{0}=\varphi_{R}$, then

$$
I=-\frac{1}{4} \eta \quad \text { and } \quad k^{2}=\frac{1}{16} \eta^{2} .
$$

Therefore, from the theorem above it follows that for $|\eta|$ larger than the critical value 4 the beating motion is forbidden (see Figure 1). In such a way, we put on a fully rigorous basis the results obtained by [19] in the two-level approximation. 


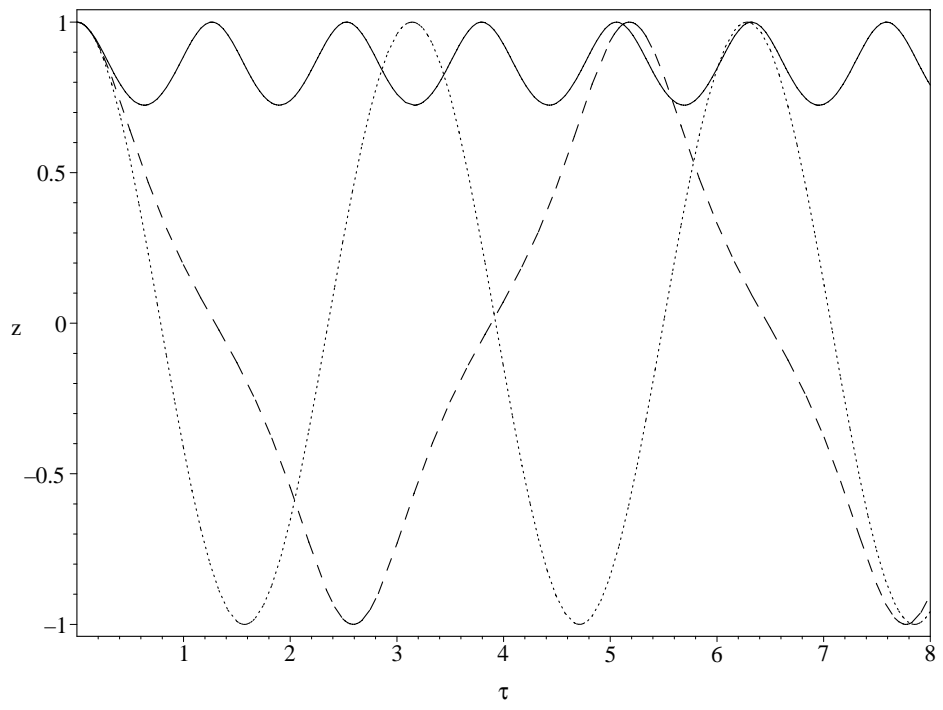

FIG. 1. Absence of the beating motion of the center of mass for nonlinearity larger than a critical value. Here, we plot the imbalance function $z(\tau)$ for different values of the nonlinearity parameter $\eta$, where $\tau=\omega t / \hbar$ denotes the slow time. For $\eta=0$ (point line) and $\eta=3.8$ (broken line) we still have a beating motion; in contrast, for $\eta$ larger than the critical value 4, e.g., $\eta=6.5$ (full line), the beating motion is forbidden.

Acknowledgment. I thank Prof. Vincenzo Grecchi and Prof. André Martinez for helpful discussions and remarks.

\section{REFERENCES}

[1] M. Abramowitz and I.A. Stegun, Handbook of Mathematical Functions with Formulas, Graphs, and Mathematical Tables, Wiley, New York, 1972.

[2] W.H. Aschbacher, J. Froehlich, G.M. Graf, K. Schnee, and M. Troyer, Symmetry breaking regime in the nonlinear Hartree equation, J. Math. Phys., 43 (2002), pp. 38793891.

[3] R. D'Agosta, B.A. Malomed, and C. Presilla, Stationary solutions of the Gross-Pitaevskii equation with linear counterpart, Phys. Lett. A, 275 (2002), pp. 424-434.

[4] F.A. Berezin And M.A. Shubin, The Schrödinger Equation, Kluwer Academic, Dordrecht, The Netherlands, 1991.

[5] F. Dalfovo, S. Giorgini, L.P. Pitavskit, and S. Stringari, Theory of Bose-Einstein condensation in trapped gases, Rev. Modern Phys., 71 (1999), pp. 463-512.

[6] G. Fibich, B. Ilan, and G. PapanicolaOU, Self-focusing with fourth-order dispersion, SIAM J. Appl. Math., 62 (2002), pp. 1437-1462.

[7] V. Grecchi, A. Martinez, and A. Sacchetti, Destruction of the beating effect for a nonlinear Schrödinger equation, Comm. Math. Phys., 227 (2002), pp. 191-209.

[8] E.M. Harrel, Double wells, Comm. Math. Phys., 75 (1980), pp. 239-261.

[9] B. Helffer and J. Sjöstrand, Multiple wells in the semi-classical limit I, Comm. Partial Differential Equations, 9 (1984), pp. 337-408.

[10] E. Hille, Lectures on Ordinary Differential Equations, Addison-Wesley, London, 1969.

[11] E.H. Lieb, R. Seiringer, and J. Yngvason, A rigorous derivation of the Gross-Pitaevskii energy functional for a two-dimensional Bose gas, Comm. Math. Phys., 224 (2001), pp. $17-31$.

[12] S. Raghavan, A. Smerzi, S. Fantoni, and S.R. Shenoy, Coherent oscillations between two weakly coupled Bose-Einstein condensates: Josephson effects, $\pi$ oscillations, and macroscopic quantum self-trapping, Phys. Rev. A, 59 (1999), pp. 620-633.

[13] M. Reed And B. Simon, Methods of Modern Mathematical Physics: IV. Analysis of Operators, Academic Press, New York, 1972. 
[14] A. SACChetTI, Nonlinear Time-Dependent Schrödinger Equations: The Gross-Pitaevskii Equation with Double-Well Potential, mp-arc 02-208, preprint, 2002.

[15] C. Sulem And P.L. Sulem, The Nonlinear Schrödinger Equation: Self-focusing and Wave Collapse, Springer-Verlag, New York, 1999.

[16] A. Soffer And M.I. Weinstein, Multichannel nonlinear scattering for nonintegrable equations, Comm. Math. Phys., 133 (1990), pp. 119-146.

[17] A. Soffer And M.I. Weinstein, Resonances, radiation damping and instability in Hamiltonian nonlinear wave equations, Invent. Math., 136 (1999), pp. 9-74.

[18] T.P. Tsai And H.T. YAU, Asymptotic dynamics of nonlinear Schrödinger equations: Resonance-dominated and dispersion-dominated solutions, Comm. Pure Appl. Math., 55 (2002), pp. 153-216.

[19] A. VARDI, On the role of intermolecular interactions in establishing chiral stability, J. Chem. Phys., 112 (2000), pp. 8743-8746.

[20] M.I. Weinstein, Nonlinear Schrödinger equations and sharp interpolation estimates, Comm. Math. Phys., 87 (1983), pp. 567-576.

[21] R. WEDER, Center manifold for nonintegrable nonlinear Schrödinger equations on the line, Comm. Math. Phys., 215 (2000), pp. 343-356. 